\title{
Mercado, Patricia y Daniel Cernas. 2015. Aportaciones a los estudios económico-administrativos. Reflexiones teóricas y evidencias empíricas. Estado de México:Universidad Autónoma del Estado de México.
}

\author{
Mercado, Patricia \& Daniel Cernas. 2015. Contributions \\ to economic-administrative Studies. Theoretical reflections \\ and empirical evidences. Estado de México: Universidad \\ Autónoma del Estado de México
}

Diana Mireya Nieto Hipólito ${ }^{1}$

Fecha de recepción: 16 de febrero del 2018

Fecha de aceptación: 16 de febrero del 2018

$\mathrm{I}$

ntegrar en un documento teorías y evidencias de estas, representa una labor nada fácil de realizar, y más cuando se trata de fundamentos relacionados con las ciencias económico administrativas, sin embargo, el libro Aportaciones a los estudios económico-administrativos. Reflexiones teóricas y evidencias empíricas lo logró. Pues agrupó en un texto científico, resultados de las reflexiones sobre la administración y la economía, concebidas desde una simbiosis de tutores, tutorados e investigadores experimentados. La necesidad de abordar la problemática presentada dentro de una empresa de manera rigurosa, requiere preparación académica colaborativa, por un lado estudiantes con un deseo de ofrecer soluciones y la experiencia de académicos. De ahí que en la mayoría de los doce capítulos en los que se divide el libro, colaboraran tutores y tutorados, investigadores, alumnos o egresados del Doctorado en Ciencias Económico-Administrativas de la Universidad Autónoma del Estado de México.

Las páginas de la obra, favorecen la comprensión de conceptos como la sustentabilidad organizacional, la competitividad, el desempeño organizacional, el aprendizaje y conocimiento organizacional, la economía del

1- Nacionalidad: Mexicana. Grado: Doctorado. Especialización: Administración. Adscripción: Universidad Tecnológica de Ciudad Juárez. Correo electrónico: dianam.nietoh@gmail.com. (D) ORCID: http://orcid.org/0000-0002-0548-3465 
conocimiento, la alianza estratégica, la rentabilidad empresarial, el mercado de trabajo y el crecimiento económico a partir de las teorías que las explican. Los enfoques teóricos incluidos en cada uno de los capítulos, fundamentan la dinámica organizacional, con el propósito de incrementar el acervo científico en el campo de las ciencias económico administrativas, al ofrecer modelos y líneas para futuras investigaciones que contribuyan a reforzar el círculo virtuoso de la competitividad y la calidad de vida de una nación.

Cabe comentar, que garantizar la confiabilidad teórica de una investigación, requiere de una profunda revisión de literatura, acompañada de fundamentos y argumentos donde se contrasta las aportaciones de más de un teórico en relación a un tópico específico. El documento que nos ocupa, sirve como guía tanto para investigadores principiantes como reconocidos, al profundizar en teorías como la Dependencia de Recursos, la Teoría de los recursos y las capacidades, la Teoría subjetiva del valor, la Teoría de los Stakeholders, la Teoría Neoclásica y la Teoría de la Segmentación por mencionar algunas.

El libro inicia con una promesa de disponibilidad de recursos, obtención de ventaja competitiva y creación de valor, por medio de un Modelo de Sustentabilidad Organizacional; la propuesta de Mercado, García y Jiménez se fundamenta en la Teoría de la Dependencia de Recursos y la Teoría de Recursos y Capacidades.

Continuando con un segundo capítulo, Demuer, Mercado y Nova exploran el Modelo de Competitividad Sistémica para ahondar en los determinantes que impulsan la competitividad empresarial, resaltando entre ellos la tecnología de la estructura productiva, el sistema de administración, el mercado, la gestión de la calidad, la gestión financiera y los sistemas de información.

De igual forma, en el tercer capítulo, Gómez y Rosales reflexionan sobre la necesidad de aprovechar el Aprendizaje Organizacional para impulsar la Competitividad Municipal; para ello sugieren la creación de ambientes propicios para el aprendizaje, un manejo adecuado de la información, fomentar la innovación y actualizar los procedimientos en los municipios. El apartado hace hincapié, en la relevancia de contar con ciudades competitivas, donde se brinde una calidad de vida a sus habitantes, posibilidades de inversión para empresas que participen en el mercado nacional e internacional y el ofrecimiento de oportunidades de empleo diversas.

Posteriormente, Machorro, Mercado y Romero consideran la aplicación del Modelo de Excelencia Administrativa de la Fundación Europea de Gestión de la Calidad (EFQM), como una alternativa para evaluar el desempeño organizacional de las universidades. Indican que la Teoría Subjetiva del Valor, la Teoría de los Stakeholders, la Teoría de la Dependencia de Recursos y la Teoría de los Recursos y Capacidades, sirven de base para explicar por qué y cómo evaluar el desempeño organizacional favorece la ventaja competitiva. Los autores apuestan por el EFQM, luego de compararlo con otros Modelos de Excelencia como el Deming, el Malcom Baldrige y el Modelo Iberoamericano de Excelencia en la Gestión de la Fundación Iberoamericana para la Gestión de la Calidad (FUNDIBEQ). Hicieron un comparativo entre sus características de enfoque, criterios y principios fundamentales.

Por otra parte, en el capítulo quinto, Canales, Godínez y González describen el conocimiento dentro del contexto de la Economía del Conocimiento, resaltando su capacidad para propiciar la innovación y el cambio tecnológico, mientras se favorece el desarrollo económico y social. Ellos proponen un marco metodológico formado por aspectos cuantitativos y cualitativos. Los primeros de ellos, conformados por los indicadores posicionales y estructurados resultado de un análisis de Redes Sociales. Por otro lado, el análisis de Redes de Conocimiento produce aspectos cualitativos como los objetivos de interacción, las características reticulares-cognitivas, mecanismos de integración, dimensiones y resultados. 
Cuando dos o más empresas combinan sus recursos para formar una tercera, conforman así una alianza estratégica, conocida como "joint venture", de esto se ocupan en el capítulo sexto Cernas, Nava y Caballero. Ellos describen como una "joint venture", permite crear valor, impactar en el desempeño financiero de una empresa e incluso lograr una ventaja competitiva, como resultado de colaborar en áreas de mercadotecnia o manufactura. Este apartado, muestra evidencia empírica de 196 joint ventures realizadas por 127 empresas estadounidenses durante el periodo comprendido del $2000 \mathrm{al} 2003$. Llama la atención un cambio en el retorno sobre los activos (ROA) después de la formación de una joint venture, los autores consideran que el efecto no varía cuando las empresas se unen con otras en el área de manufactura o mercadotecnia.

En relación al mercado de trabajo, Rubio y Salgado en el capítulo séptimo, explican este tópico desde la óptica de la Teoría Neoclásica y la Teoría de la Segmentación. En la primera consideran los supuestos del mercado de trabajo y aquellos relacionados con la ley de la oferta y la demanda, además de integrar la Teoría del Capital Humano. Con respecto a la Teoría de la Segmentación, incluyen una descripción de sus generaciones, agrupándolas de la siguiente manera: primera generación la Teoría Institucionalista, segunda generación la Teoría Radical, tercera generación la Teoría Multicausal y cuarta generación Toyotismo.

El libro también incluye, una descripción del rol institucional del gobierno en dependencia de recursos naturales. En el capítulo octavo, Banegas y González dan respuesta a dos interrogantes, la primera de ellas cuestiona el rol del gobierno en países dependientes de recursos naturales, mientras que la segunda pregunta considera la imperante necesidad de implementar una regla fiscal para la protección de los mismos. Durante el desarrollo del capítulo, se propone una política fiscal para blindar el gasto público.

Continuando con temas económicos, Nájera y Agüero en el capítulo noveno, parten del supuesto que el precio de la opción sobre el tipo de cambio peso mexicano-dólar estadounidense otorga un precio más justo cuando se consideran los efectos inesperados del mercado en la volatilidad estocástica. Para probar su hipótesis, adaptaron el Modelo de Volatilidad Estocástica de Heston (1993), investigaron su desempeño en relación con el Modelo de Black-Scholes (1973) y posteriormente exploraron el proceso de tipo de cambio en la estimación de los parámetros del Modelo de Heston (1993). Este apartado, incluye una descripción de los Modelos de Opción, además de una explicación de los Modelos de precios de opciones sobre el tipo de cambio.

Por otro lado, Rendón y Salgado, en el capítulo décimo, explican las causas del crecimiento económico regional, enfocándose en la oferta y demanda. Para su análisis de la oferta, revisaron las teorías neoclásicas del crecimiento endógeno, el progreso técnico así como la especialización y crecimiento. Mientras al examinar la demanda, se fundamentan en las Teorías de Crecimiento, especialmente en los Modelos Keynesianos de Myrdall (1957). También hacen referencia a las Leyes de Kaldor y la Ley de Verdoorn.

En el capítulo onceavo, Munguía, Quiroz y Martínez describen de manera cronológica el papel de la Inversión Extranjera Directa (IED), desde finales del siglo XVIII hasta el 2010. Esto sirve como antecedente, para conceptualizar la IED, resaltando su importancia en la competitividad regional, nacional y empresarial. Para fundamentar la relación teórica entre la IED y la competitividad, los autores recurren a los Modelos de Competitividad Sistémica, Estructural y Sectorial. De igual forma, describen los enfoques microeconómico y macroeconómico de la IED y la competitividad. También, incluyen algunas teorías sobre los determinantes de la IED, entre las que se encuentran las teorías tradicionales del comer- 
- Revista de Ciencias Sociales y Humanidades. ISSN-P: 0188-9834 ISSN-E:2395-8669.

cio, como la Teoría de la Organización Industrial, la Teoría de la Internalización, la Teoría ecléctica de Dunning, la Teoría japonesa de Kojima y la Teoría de Localización de la IED. El capítulo cierra con un análisis de los factores de la IED y la competitividad.

Finalmente, Brito en el capítulo doceavo, explica teóricamente la dinámica del empleo y la desocupación; fundamenta sus argumentos en la Teoría general de la ocupación, el interés y el dinero de Keynes. Durante su explicación, se examinan distintas corrientes del pensamiento keynesiano, incluso se aprecian algunas discrepancias entre ellos. Este apartado, incluye una descripción del modelo keynesiano para mostrar la demanda de consumo y la demanda de inversiones privadas. Luego apunta a la corriente postkeynesiana y neokeynesiana.

Las líneas del libro Aportaciones a los estudios económico-administrativos. Reflexiones teóricas y evidencias empíricas, permiten al lector contrastar la realidad de la dinámica organizacional en los sectores público y privado, al tiempo de ofrecer soluciones estratégicas para mejor su desempeño. En cada uno de los capítulos, se asoman la ventaja competitiva y la competitividad, resaltando el impacto positivo que está última tiene en una nación.

Sirva la presente reseña, para felicitar a los colaboradores y coordinadores por el esfuerzo realizado, sus aportaciones a la comunidad científica y en específico al contexto de las ciencias económico administrativas. Además, se invita a aquellos interesados en aportar conocimiento teórico y evidencias empíricas en esta área de la ciencia a examinar sus páginas con detenimiento. 